\title{
Earlier Return to Light Duty Is Associated With Successful Return to Full Duty of Workers' Compensation Patients Treated With Shoulder Arthroscopic Surgery
}

\author{
Patrick A. Massey, M.D., Gabriel Sampognaro, M.D., Phillip Fincher, B.S., \\ Samantha Vance, B.S., Milan Mody, M.D., and R. Shane Barton, M.D.
}

\begin{abstract}
Purpose: To evaluate Workers' Compensation (WC) patients who underwent arthroscopic shoulder surgery for workplace shoulder injuries and to determine whether there was an association between earlier return to light duty and earlier return to full duty. Methods: After receiving institutional review board approval, we performed a retrospective chart review of all WC patients treated with shoulder arthroscopic surgery by 2 senior authors between 2011 and 2018. The patients were divided into 2 groups: Group 1 went back to light-duty work within the first 100 days after surgery, whereas group 2 performed light-duty work after 100 days or performed no light-duty work. The primary outcomes included the length of time from surgery to light-duty work and the length of time from surgery to return to the full-duty work level. Results: A total of 59 patients met the inclusion criteria. There was a moderate correlation between the number of days at which the patients were released to light duty and the days they were able to be released to full duty $(r=0.35)$. In group 1 (light duty $\leq 100$ days), 18 patients $(75 \%)$ went back to full duty, whereas only 16 patients $(46 \%)$ in group 2 were able to return to full-duty work $(P=.025)$. Conclusions: Earlier return to light duty is associated with earlier return to full duty after shoulder arthroscopic surgery in patients with a Workers' Compensation claim. Additionally, WC patients who returned to early light duty in the first 100 days postoperatively had a higher rate of return to full duty than did patients who did not return to early light duty. Level of Evidence: Level III, case-control study.
\end{abstract}

$\mathbf{I}$ $\mathrm{n}$ the workplace, physical labor has often been associated with trauma and injury to the rotator cuff of the shoulder with subsequent shoulder pain. ${ }^{1}$ Rotator cuff injury and pain comprise the second most common workplace injury behind back and neck injury. ${ }^{l}$ Injuries to the shoulder cause a significant

From the Department of Orthopaedic Surgery, Louisiana State University, Shreveport, Louisiana, U.S.A. (P.A.M., G.S., S.V., R.S.B.); School of Medicine, Louisiana State University, Shreveport, Louisiana, U.S.A. (P.F.); and WillisKnighton Health System, Shreveport, Louisiana, U.S.A. (M.M.).

The authors report no conflicts of interest in the authorship and publication of this article. Full ICMJE author disclosure forms are available for this article online, as supplementary material.

Received October 11, 2021; accepted January 11, 2022

Address correspondence to Patrick A. Massey, M.D., Department of Orthopaedic Surgery, Louisiana State University, 1501 Kings Hwy, Shreveport, LA 71103,U.S.A.E-mail: Patrick.massey@lsuhs.edu

(C) 2022 THE AUTHORS. Published by Elsevier Inc. on behalf of the Arthroscopy Association of North America. This is an open access article under the CC BY-NC-ND license (http://creativecommons.org/licenses/by-nc-nd/4.0/). 2666-061X/211430

https://doi.org/10.1016/j.asmr.2022.01.004 decrease in the quality of life in patients affected, leading to an overall decrease in their workplace productivity among other effects. ${ }^{1}$ Patients will often undergo conservative treatment seeking relief, and when this is unsuccessful, they may be treated surgically to improve joint function and decrease pain, leading to an increased quality of life. ${ }^{2-4}$

Injuries to the upper extremity, including the shoulder, account for a large portion of missed workdays. ${ }^{5}$ Depending on the patient's employer, the patient may or may not receive compensation for his or her injuries. Workers' Compensation (WC) patients present a higher cost to health care systems on average and account for a total of $12 \%$ of payments to some orthopaedic clinics. ${ }^{5}$ For many reasons, WC patients are more frequently associated with poorer outcomes after shoulder arthroscopy than non-Workers' Compensation (NWC) patients after the same procedure or a similar procedure. ${ }^{1}$ One study reported that $42 \%$ of WC patients achieved good to excellent outcomes whereas $72 \%$ of NWC patients achieved good to excellent outcomes. ${ }^{6}$ The inferior surgical outcomes associated with WC 
may lead to a lower rate of return to work (RTW) especially in the heavy-work population, which involves lifting substantial loads over the head. ${ }^{7}$ Moreover, when comparing average RTW time, several studies have noted that WC patients take a longer amount of time to return to their work on average when compared with NWC patients. ${ }^{8-10}$ Another study, examining rotator cuff repair outcomes in 100 patients, reported that WC patients were less likely to RTW. ${ }^{11}$ Balk et al. ${ }^{12}$ reported that although most WC and NWC patients returned to work, a higher percentage of WC patients left their jobs because of more persistent symptoms and higher reported pain levels.

Although it is well established that WC patients have a lower rate of RTW and take longer to RTW, it is important to understand the factors delaying or preventing their RTW. Several factors including age, comorbidities, workload, mechanism of injury, and surgical approach have all been linked to an increased RTW time in WC patients. ${ }^{1,13,14}$ WC patients often report a lower rate of satisfaction and recovery when compared with NWC patients. ${ }^{1}$ They also report higher rates of pain and disability, including preoperatively. ${ }^{1}$ When examining the link between RTW and shoulder surgery, Shields et al. ${ }^{15}$ found that the minimization of legal involvement and a more efficient rehabilitation during RTW may help patients RTW more quickly while reducing the overall health care costs.

Factors such as ongoing litigation may be involved in the biopsychosocial health care model of postoperative recovery. As part of this model, it has been described that self-efficacy is important in the recovery after total knee arthroplasty. ${ }^{16}$ Additional studies have reported that some work environments can positively affect well-being and even contribute to illness recovery. ${ }^{17,18}$ Although most patients who have undergone arthroscopic shoulder surgery are not able to RTW immediately, many of them can return to some form of light duty soon after surgery. ${ }^{8,19}$ The purposes of our study were to evaluate WC patients who underwent arthroscopic shoulder surgery for workplace shoulder injuries and to determine whether there was an association between earlier return to light duty and earlier return to full duty. Our hypothesis for this study was that WC patients with earlier return to light-duty work would have a higher rate of return to full duty.

\section{Methods}

This retrospective study was approved by the institutional review board. We (P.M. and G.S.) first performed a chart review of all WC patients treated surgically by the 2 senior authors between 2011 and 2018. The initial chart review was performed to determine which patients had undergone arthroscopic shoulder surgery. A search was performed for WC patients who underwent surgery during the aforementioned period with Current
Procedural Terminology (CPT) codes 29806, 29807, 29819, 29820, 29821, 29822, 29823, 29824, 29825 , 29826, 29827, and 29828. The established exclusion criteria for this study were patients with a fracture, patients undergoing revision shoulder surgery after a prior operation, and polytrauma patients treated with surgical procedures in other areas. Patients were followed up for 2 years postoperatively.

For this study, we defined "light duty" as a return of the patient back to his or her place of employment with restrictions including no use or low use (restricted range of motion and weight limits) of the postoperative extremity and lifting restrictions. We defined "full duty" as a return of the patient to his or her preinjury level of work responsibility in the same job. On review, 2 groups were established based on the amount of time before the patient went back to light duty. The early light duty (ELD) group (group 1) consisted of WC patients who went back to light duty within the first 100 days after surgery. The non-ELD group (group 2) consisted of WC patients who did not go back to light duty within 100 days after surgery.

Patient information collected for the study was as follows: age, occupation, mechanism of action of the injury, and side of the body that was injured. Primary and secondary procedures were also noted in this study, including rotator cuff repair, acromioclavicular reconstruction, biceps tenodesis, capsulorrhaphy, SLAP tear repair, subacromial decompression, distal clavicle excision, and removal of loose bodies. Jobs were classified as heavy, medium, or light according to U.S. Bureau of Labor Statistics guidelines (https://www.bls.gov/ors/ factsheet/strength.htm). ${ }^{20}$

Through a chart review, data were collected on the date of injury, date of surgery, date of actual return to light duty, and date of return to full duty. Work status was determined via work notes and work release notes. If patients had not returned to work by the 2-year postoperative date, they were deemed to have no RTW.

\section{Statistics}

The correlation between time to return to light duty and time to return to full duty was analyzed with the Pearson correlation coefficient. The types of shoulder surgery and demographic characteristics in group 1 versus group 2 were compared using the $\chi^{2}$ test. The proportion of patients who were able to return to full duty in group 1 versus group 2 was evaluated using the $\chi^{2}$ test.

\section{Results}

A total of 59 patients met the final inclusion criteria (Fig 1). Of these 59 patients, $53(90 \%)$ were able to return to some form of work; however, only $34(58 \%)$ were able to return to full duty in the same job. The average age of all patients at the time of surgery was 


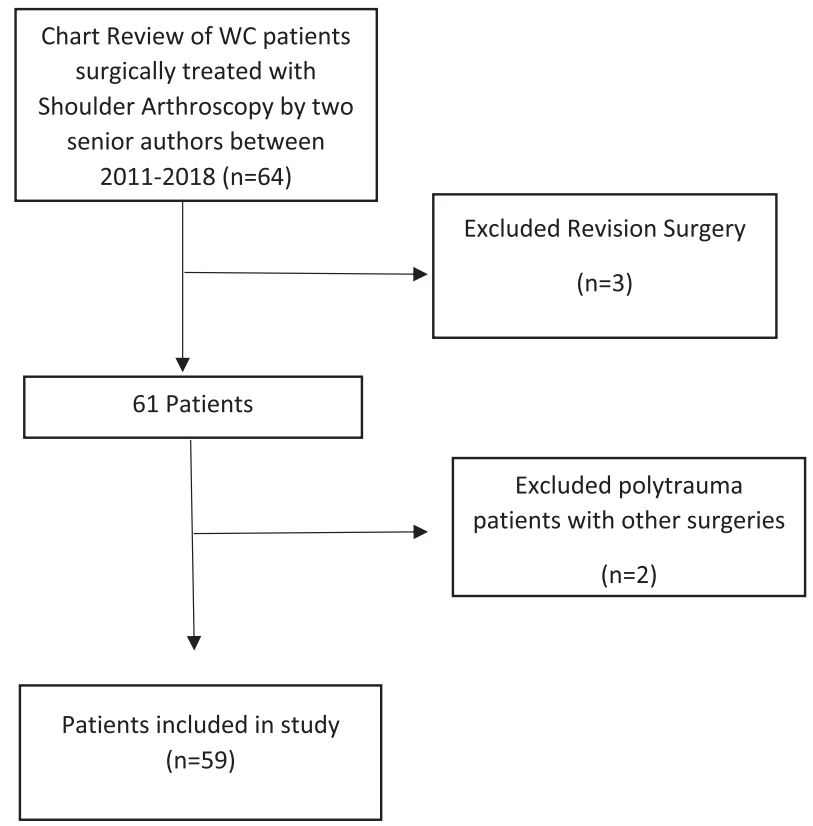

Fig 1. Flowchart showing inclusion criteria after patients were excluded for revision surgery, polytrauma with multiple other operations, and fractures. (WC, Workers' Compensation.)

$50.8 \pm 12.6$ years. There were 18 male and 41 female patients. There were no significant differences in the demographic characteristics between the ELD and nonELD groups (Table 1). There was also no statistically significant difference in the types of surgical procedures performed in the ELD group versus the non-ELD group $(P=.33)$ (Table 2$)$. There were 115 additional procedures performed, with no difference in the distribution between the ELD group and non-ELD group (Table 3). Overall, the strength demands of the patients' jobs were high in $32 \%$, medium in $53 \%$, and low in $15 \%$ (Table 4 ). There was no significant differences in the strength demands between the ELD and non-ELD groups $(P=.609)$.

We observed a moderate correlation between the number of days at which the patients were released to light duty after shoulder surgery and the number of days at which patients were able to be released to full duty after surgery $(r=0.35)$ (Fig 2$)$. Of the 24 patients in group 1 (light duty $\leq 100$ days), $16(75 \%)$ went back to full duty, whereas only 16 of the 35 patients in group $2(46 \%)$ were able to return to full-duty work by the 2year endpoint. The rate of return to full duty was significantly higher in patients who returned to light duty within 100 days $(P=.025)$. For all patients, the average number of days to return to full duty was $194 \pm$ 87 days among those who were able to fully return.

In addition to comparing the association of ELD and return to full duty, we compared the effects of factors such as the type of surgery and hand dominance on rates of return to full duty. When we compared the types of primary surgery for full RTW, $44 \%$ of the patients who underwent shoulder instability repair returned to full duty whereas $65 \%$ of those who underwent rotator cuff repair were able to return to full duty. There was no significant difference in the rate of return to full duty based on the type of primary procedure among all patients $(P=.237)$. Hand dominance was also evaluated, with $84 \%$ of patients being right hand dominant and $16 \%$ being left hand dominant. In the ELD group, $80 \%$ of patients underwent surgery on the dominant-hand side, whereas in the non-ELD group, $57 \%$ underwent surgery on the dominanthand side, with no significant difference between groups $(P=.129)$. Patients who underwent surgery on the dominant-hand side had a 55\% return to full duty, whereas those with surgery on the nondominant side had a $65 \%$ return to full duty. Surgery on the dominant-hand side versus the nondominant side was not associated with a higher rate of return to full duty $(P=.557)$.

\section{Discussion}

Our study showed that a return to earlier light duty was associated with an earlier return to full duty in WC patients who underwent shoulder arthroscopy. Additionally, we found that patients who returned to light duty in the first 100 days after shoulder arthroscopy (ELD) had a higher rate of return to full duty. On the basis of these findings, orthopaedic surgeons should consider a return to ELD for WC patients after shoulder arthroscopy because it seems to be associated with an earlier return to full duty. These data suggest that employers and physicians should be communicating and collaborating to find safe light-duty positions for WC patients to continue to heal in the workplace before being assigned back to full duty.

WC is intended to financially compensate injured employees during the recovery period so that they can RTW at the earliest possible time. ${ }^{21,22}$ Individuals who remain out of work for extended periods will often never return to the workforce, and those who first return to light duty will often have faster recovery

Table 1. Demographic Characteristics of Shoulder Arthroscopy Workers' Compensation Patients Who Returned to ELD and Who Did Not Return to ELD

\begin{tabular}{lrcc}
\hline & ELD Group & Non-ELD Group & $P$ Value \\
\hline $\begin{array}{l}\text { Age, mean (SD), yr } \\
\text { Sex, n (\%) }\end{array}$ & $52.6(14.0)$ & $49.5(11.5)$ & .36 \\
$\quad$ Male & $9(37.5)$ & $9(25.7)$ & \\
$\quad$ Female & $15(62.5)$ & $26(74.3)$ & .40 \\
Side, n (\%) & & & \\
$\quad$ Left & $8(33.3)$ & $13(37.1)$ & \\
$\quad$ Right & $16(66.7)$ & $22(62.9)$ & .79 \\
\hline
\end{tabular}


Table 2. Primary Shoulder Surgery for Workers' Compensation Patients Who Returned to ELD and Who Did Not Return to ELD

\begin{tabular}{lccr}
\hline & ELD Group & Non-ELD Group & Total \\
\hline Procedure, n & & 1 & \\
AC reconstruction & 1 & 7 & 2 \\
Bankart repair & 2 & 2 & 9 \\
Biceps treatment & 1 & 0 & 3 \\
Biceps tenodesis & 1 & 18 & 1 \\
Rotator cuff repair & 18 & 5 & 36 \\
Capsular release & 1 & 1 & 6 \\
DCE & 0 & 0 & 1 \\
SAD & 1 & 0 & 1 \\
Tenotomy & 1 & 35 & 1 \\
Total & 25 & 60 \\
\hline NOTE. No difference in the types of primary shoulder surgical \\
procedures was observed between the 2 groups (P =.33). \\
AC, acromioclavicular; DCE, distal clavicle excision; ELD, early light \\
duty; SAD, subacromial decompression.
\end{tabular}

times. ${ }^{21,22}$ Some places of employment offer light-duty work as well as alternative work opportunities for their injured employees to return to the workplace more quickly. ${ }^{21}$ The most common RTW offer is light duty, which is typically less physically demanding and allows the injured employee to reintegrate back into his or her place of employment in a more comfortable and less stressful setting than with full-duty responsibilities. $^{21,23}$ Many employers are even able to withdraw WC from an injured employee with just cause if that employee declines to accept a light-duty work offer. ${ }^{21}$

Although some studies report on RTW, it is important to delineate RTW in some form versus a return to full duty in the same job. Previous studies have not always shown a high rate of return to full duty. ${ }^{71}{ }^{19}$ Imai et al. ${ }^{7}$ found that only $66.7 \%$ of patients were able to return to full duty after rotator cuff repair. Nové-Josserand et al. ${ }^{19}$ found that almost $60 \%$ of patients treated with rotator cuff repair for work-related injuries returned to work in the same position or a similar position to the position they held before injury and $16 \%$ of patients did not RTW at all as a direct result of shoulder injury. The type of work was found to have little or no impact in determining the eventual RTW, but it did significantly impact the length of time to return work. ${ }^{19}$ The $60 \%$ RTW rate presented by Nové-Josserand et al. mirrors the findings of our study, in which overall, only $58 \%$ of patients returned to full duty in the same job. In addition, similar to their finding that $16 \%$ of patients had no RTW at all, our data showed that $10 \%$ of patients were unable to RTW at all.

Previous studies have reported on the mean length of time for WC patients to return to full duty compared with NWC patients after shoulder surgery. ${ }^{7,24}$ These studies have shown that WC patients have worse
Table 3. Additional Surgical Procedures for Workers' Compensation Patients Who Returned to ELD and Who Did Not Return to ELD

\begin{tabular}{lrcrr}
\hline & $\begin{array}{c}\text { ELD } \\
\text { Group }\end{array}$ & $\begin{array}{c}\text { Non-ELD } \\
\text { Group }\end{array}$ & Total & $P$ Value \\
\hline Procedure, $\mathrm{n}$ & & & & \\
$\quad$ Biceps tenodesis & 15 & 17 & 32 & .425 \\
SAD & 16 & 26 & 42 & .395 \\
DCE & 6 & 13 & 19 & .402 \\
Capsulorrhaphy & 2 & 2 & 4 & $>.999$ \\
AC reconstruction & 1 & 0 & 1 & .407 \\
SLAP repair & 4 & 6 & 10 & $>.999$ \\
ROLB & 0 & 0 & 0 & $>.999$ \\
Tenotomy & 1 & 1 & 2 & $>.999$ \\
Loose body treatment & 1 & 2 & 3 & $>.999$ \\
Capsular release & 0 & 2 & 2 & .509 \\
Total & 46 & 69 & 115 &
\end{tabular}

AC, acromioclavicular; DCE, distal clavicle excision; ELD, early light duty; ROLB, removal of loose bodies; SAD, subacromial decompression.

outcomes, take longer to RTW, and are at an increased risk of no return to full duty. A variety of factors have been shown to be associated with worse outcomes among WC patients, such as active litigation and job demands. ${ }^{13,15,25-27}$ Some places of employment offer alternative work environments including light-duty work offers to WC patients to help these patients RTW more quickly and improve outcomes. ${ }^{21,22}$ However, there are still many places of employment that do not offer sufficient RTW options such as light duty or that only allow their WC patients to return to full duty. ${ }^{21,22}$ These facts suggest the importance of light duty and the role it may play in contributing to a successful return to full duty. ${ }^{21}$

Injured employees who have sought WC may have a low incentive to RTW because they receive around two-thirds of their normal wage while on WC. ${ }^{28}$ The Americans With Disabilities Act placed a greater responsibility on employers by requiring them to offer workplace accommodations to both disabled employees and employees who have been injured and wish to return to the workplace. ${ }^{28}$ Light duty is one of the most recommended workplace accommodations among case managers for returning $\mathrm{WC}$ patients to the

Table 4. Occupational Strength Levels for Workers' Compensation Patients Who Returned to ELD and Who Did Not Return to ELD

\begin{tabular}{lccc}
\hline Strength & & & \\
Demand & ELD Group, n (\%) & Non-ELD Group, n (\%) & P Value \\
\hline Low & $5(21)$ & $4(11)$ & .609 \\
Medium & $12(50)$ & $19(54)$ & \\
High & $7(29)$ & $12(34)$ & \\
\hline
\end{tabular}

ELD, early light duty. 
Days from Surgery to Light Duty Versus Days from Surgery to Full Duty

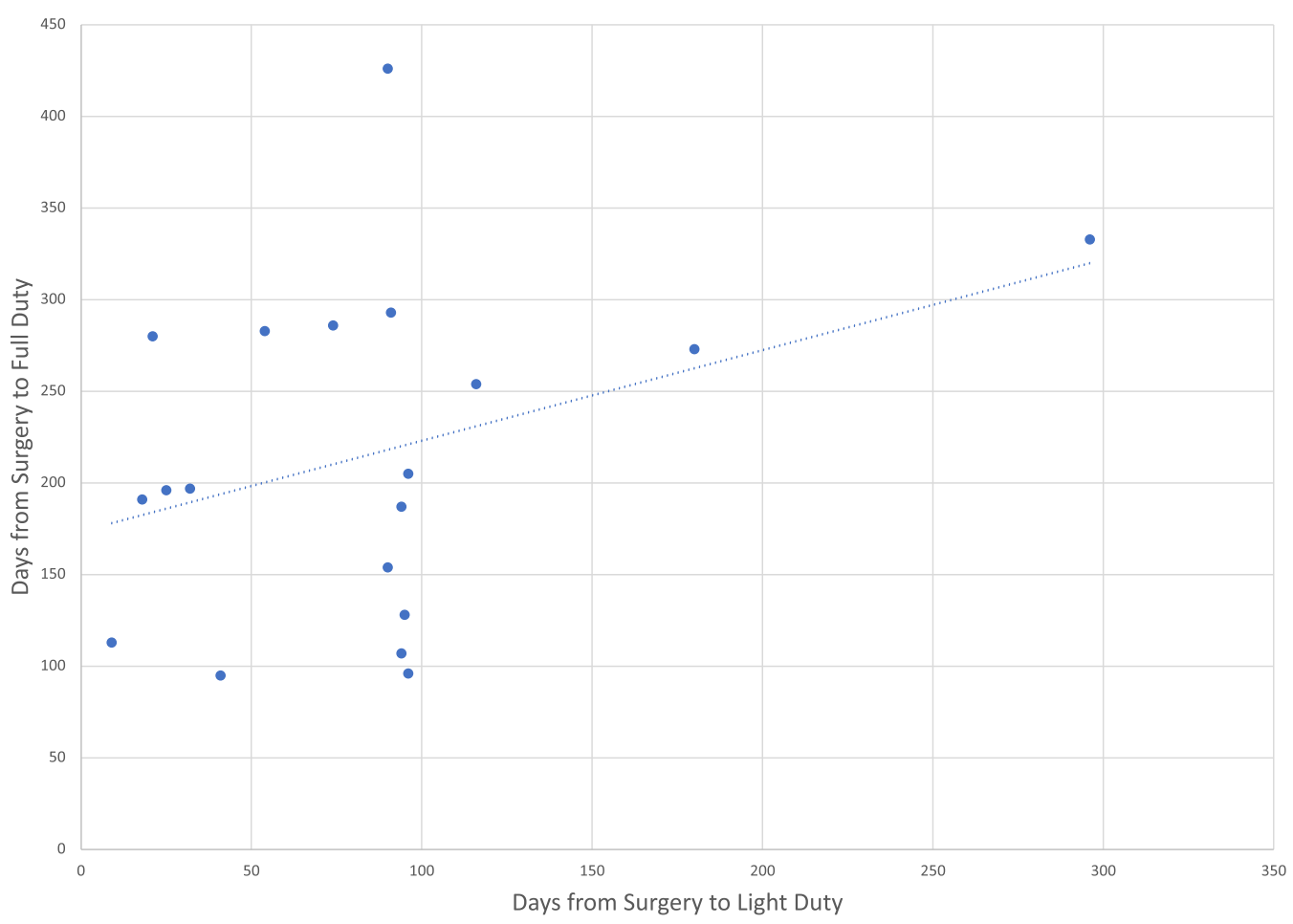

Fig 2. Scatter plot diagram showing association between earlier return to light duty and earlier return to full duty. A moderate association $(r=0.35)$ was found.

workplace quickly. ${ }^{23}$ This allows a physician to lay out parameters for the injured employee to follow while the employee re-enters the workplace in a modified version of his or her preinjury job or in a completely different job that may be specially created for the injured employee. ${ }^{29}$ Setting up light-duty accommodations allows injured employees, including WC patients, to continue to heal in the workplace with restrictions and limitations in place to help implement an acceptable workload for these patients with minimal injury risk. ${ }^{22}$ Employers that offer light-duty accommodation often see an eventual return to full duty as well as faster recovery times among WC patients. ${ }^{21,22,24}$

There may be positive psychological and physical benefits to returning to work, even in a light-duty capacity. Several studies have analyzed some of the factors contributing to good outcomes after surgical intervention for shoulder injuries. In a study that analyzed the impact of emotional health on outcomes after various orthopaedic procedures, Ayers et al. ${ }^{16}$ found that patients with higher emotional well-being had better surgical outcomes than patients with poor emotional well-being. Being employed has also been linked to good mental health if the place of employment has "good mental health practices" such as social interactions and good supervision. ${ }^{17,18}$ Employers should be encouraged to display these "good practices" by the orthopaedic surgeon, as well as to provide lightduty work for WC patients. In addition, self-efficacy has been associated with a higher rate of RTW. ${ }^{25}$ It was also found that being employed in a good psychosocial work environment leads to good mental health and increased employment rates. ${ }^{18}$ It may be that ELD or earlier RTW in some form has a positive effect on a patient's view of his or her recovery process, gaining higher self-efficacy and benefit from a positive psychosocial work environment, leading to a higher rate of return to full duty.

Patient behaviors including malingering and false exaggeration of disability must also be considered when analyzing the time between return to light duty and subsequent return to full duty in WC patients. Bianchini et al. ${ }^{30}$ reviewed 332 patients with traumatic brain injury and determined that $30 \%$ to $40 \%$ of WC claims were being falsely exaggerated for a variety of reasons. On further investigation, the primary motivating factor behind the exaggeration of disability was found to be secondary gain in the form of disability payments. ${ }^{31}$ A secondary motivating factor was found to be another type of personal gain in the form of time off from work. ${ }^{5}$ These findings suggest that WC patients often view their WC payments and time off as superior alternatives to returning to their job responsibilities. Removal of these motivating factors may be accomplished by ELD, in that patients are now receiving paychecks as opposed to disability payments. 
Proper rehabilitation and patient education also likely play an important role in determining how a patient reintegrates back into the workplace. There has been moderate evidence showing that pain and avoidance behavior were predictive of inferior RTW outcomes, which are thought to result from the patient believing that he or she has a higher level of disability than is actually present. ${ }^{32}$ Godges et al. ${ }^{33}$ stated that educating the patient on proper pain management techniques and methods to remain physically active helped to reduce the number of days before returning to full duty. This finding suggests that physical therapy is necessary not only to provide strengthening and mobility of the shoulder postoperatively but also to lessen the impact of pain and avoidance behaviors on patients while they are becoming accustomed to using the shoulder in the workplace. When combined with our findings about return to ELD, physical therapy and proper pain management education likely play an important role in a quicker return to full duty among WC patients after shoulder surgery.

Although our study showed that ELD was associated with a quicker and higher likelihood of return to full duty, there are other factors that were not related. Our study showed that the type of surgery was not related to return to full duty. Additionally, surgery on the dominant-hand side was not associated with return to full duty.

Our study retrospectively reviewed the charts of WC patients who underwent shoulder arthroscopy between 2011 and 2018 and set out to determine whether a return to light duty within 100 days of the shoulder arthroscopy resulted in a quicker return to full duty. The rate of return to full duty was significantly higher among WC patients who returned to light duty within 100 days of shoulder arthroscopy. The speed at which the patients return to light duty is one of several factors affecting return to full duty, including psychological, psychosocial, socioeconomic, and physical contributions. On the basis of this information, we believe it is important for the physician and the employer to collaborate with one another to create a plan for WC patients to return to light duty so that they can return to their preinjury work responsibilities and quality of life more quickly and have a higher chance of successful RTW.

\section{Limitations}

This study is not without limitations. There were geographic limitations in that we only included patients from one community hospital, and we did not use Current Procedural Terminology (CPT) codes to include WC patients from across the country. Another limitation of this study was the use of cases treated by 2 experienced surgeons who have expertise in shoulder surgery. Thus, our results may not correlate with the results of other orthopaedic surgeons with different levels of expertise from whom WC patients may often seek medical treatment. There was also no standardization of clinical outcome in this study based on perioperative and intraoperative decisions because we were dealing with 2 separate surgeons and thought processes.

Our study included a diverse pathology pool with differing time frames of healing and returning to full activity. As a result, it is possible that some patients with more severe pathology could have had extended recovery times or required a change in employment owing to limitations and complications of a more severe injury. We included different types of shoulder arthroscopic surgery because independent of the type of surgery, it is considered safe to return patients to light duty after arthroscopic shoulder surgery. Our data show that there was no difference between groups regarding the types of operations, lowering the likelihood of selection bias. Another potential limitation involves patients who chose to retire instead of returning to work, leading to confounding data on ELD and RTW.

\section{Conclusions}

Earlier return to light duty is associated with earlier return to full duty after shoulder arthroscopic surgery in patients with a WC claim. Additionally, WC patients who returned to ELD in the first 100 days postoperatively had a higher rate of return to full duty than did patients who did return to ELD.

\section{Acknowledgment}

The authors acknowledge Amanda Hunt for preparation of the manuscript and Andreas Chen for assistance with development of the project.

\section{References}

1. Balyk R, Luciak-Corea C, Otto D, Baysal D, Beaupre L. Do outcomes differ after rotator cuff repair for patients receiving workers' compensation? Clin Orthop Relat Res 2008;466:3025-3033.

2. Buess E, Steuber KU, Waibl B. Open versus arthroscopic rotator cuff repair: A comparative view of 96 cases. Arthroscopy 2005;21:597-604.

3. Mather RC, Koenig L, Acevedo D, et al. The societal and economic value of rotator cuff repair. J Bone Joint Surg Am 2013;95:1993-2000.

4. MacDermid JC, Ramos J, Drosdowech D, Faber K, Patterson S. The impact of rotator cuff pathology on isometric and isokinetic strength, function, and quality of life. J Shoulder Elbow Surg 2004;13:593-598.

5. Day CS, Alexander M, Lal S, et al. Effects of workers' compensation on the diagnosis and surgical treatment of patients with hand and wrist disorders. J Bone Joint Surg Am 2010;92:2294-2299. 
6. Essman JA, Bell RH, Askew M. Full-thickness rotator-cuff tear. An analysis of results. Clin Orthop Relat Res 1991;265: $170-177$.

7. Imai T, Gotoh M, Hagie K, et al. Factors affecting return to work in patients undergoing arthroscopic rotator cuff repair. Prog Rehabil Med 2019;4:20190006.

8. Nicholson GP. Arthroscopic acromioplasty: A comparison between workers' compensation and non-workers' compensation populations. J Bone Joint Surg Am 2003;85: 682-689.

9. Aagaard KE, Randeblad P, Abu-Zidan FM, Lunsjö K. Return to work after early repair of acute traumatic rotator cuff tears. Eur J Trauma Emerg Surg 2020;46:817-823.

10. Carmona L, Faucett J, Blanc PD, Yelin E. Predictors of rate of return to work after surgery for carpal tunnel syndrome. Arthritis Care Res 1998;1 1:298-305.

11. Hawkins R, Misamore G, Hobeika P. Surgery of fullthickness rotator-cuff tears. J Bone Joint Surg Am 1985;67:1349-1355.

12. Balk ML, Hagberg WC, Buterbaugh GA, Imbriglia JE. Outcome of surgery for lateral epicondylitis (tennis elbow): Effect of worker's compensation. Am J Orthop (Belle Mead NJ) 2005;34:122-126, discussion 126.

13. Yamamoto A, Takagishi K, Osawa T, et al. Prevalence and risk factors of a rotator cuff tear in the general population. J Shoulder Elbow Surg 2010;19:116-120.

14. Henn RF, Kang L, Tashjian RZ, Green A. Patients with workers' compensation claims have worse outcomes after rotator cuff repair. J Bone Joint Surg Am 2008;90:2105-2113.

15. Shields E, Thirukumaran C, Noyes K, Voloshin I. A review of a workers' compensation database 2003 to 2013: Patient factors influencing return to work and cumulative financial claims after rotator cuff repair in geriatric workers' compensation cases. Geriatr Orthop Surg Rehabil 2017;8:208-214.

16. Ayers DC, Franklin PD, Ring DC. The role of emotional health in functional outcomes after orthopaedic surgery: Extending the biopsychosocial model to orthopaedics. J Bone Joint Surg Am 2013;95:e165.

17. Modini M, Joyce S, Mykletun A, et al. The mental health benefits of employment: Results of a systematic meta-review. Australas Psychiatry 2016;24:331-336.

18. Butterworth P, Leach LS, Strazdins L, Olesen SC, Rodgers B, Broom DH. The psychosocial quality of work determines whether employment has benefits for mental health: Results from a longitudinal national household panel survey. Occup Environ Med 2011;68:806-812.

19. Nové-Josserand L, Liotard JP, Godeneche A, et al. Occupational outcome after surgery in patients with a rotator cuff tear due to a work-related injury or occupational disease. A series of 262 cases. Orthop Traumatol Surg Res $2011 ; 97: 361-366$.

20. U.S. Bureau of Labor Statistics. Strength levels. https:// www.bls.gov/ors/factsheet/strength.htm.

Accessed

October 4, 2021

21. Presmanes GT. Workers' compensation, return to work, and use of light duty work offers: An overview of programs throughout the United States. Tort Trial Insur Pract Law J 2015;50:781-808.

22. Bible JE, Spengler DM, Mir HR. A primer for workers' compensation. Spine J 2014;14:1325-1331.

23. Lincoln AE, Feuerstein M, Shaw WS, Miller VI. Impact of case manager training on worksite accommodations in workers' compensation claimants with upper extremity disorders. J Occup Environ Med 2002;44:237-245.

24. Bhatia S, Piasecki DP, Nho SJ, et al. Early return to work in workers' compensation patients after arthroscopic fullthickness rotator cuff repair. Arthroscopy 2010;26:1027-1034.

25. Cancelliere C, Donovan J, Stochkendahl MJ, et al. Factors affecting return to work after injury or illness: Best evidence synthesis of systematic reviews. Chiropr Man Therap 2016;24:32.

26. Cho CH, Seo HJ, Bae KC, Lee KJ, Hwang I, Warner JJP. The impact of depression and anxiety on self-assessed pain, disability, and quality of life in patients scheduled for rotator cuff repair. J Shoulder Elbow Surg 2013;22:1160-1166.

27. Largacha M, Parsons IM IV, Campbell B, Titelman RM, Smith KL, Matsen F. Deficits in shoulder function and general health associated with sixteen common shoulder diagnoses: A study of 2674 patients. J Shoulder Elbow Surg 2006;15:30-39.

28. Kamer GJ, DeSouza BD. The light duty dilemma. Labor Lawyer 1996;12:247-260.

29. Krause N, Dasinger LK, Neuhauser F. Modified work and return to work: A review of the literature. J Occup Rehabil 1998;8:113-139.

30. Bianchini KJ, Curtis KL, Greve KW. Compensation and malingering in traumatic brain injury: A dose-response relationship? Clin Neuropsychol 2006;20:831-847.

31. Ghori AK, Chung KC. A decision-analysis model to diagnose feigned hand weakness. J Hand Surg Am 2007;32: 1638-1643.

32. Iles RA, Davidson M, Taylor NF. Psychosocial predictors of failure to return to work in non-chronic non-specific low back pain: A systematic review. Occup Environ Med 2008;65:507-517.

33. Godges JJ, Anger MA, Zimmerman G, Delitto A. Effects of education on return-to-work status for people with fearavoidance beliefs and acute low back pain. Phys Ther 2008;88:231-239. 